\title{
SITUAÇÃO EPIDEMIOLÓGICA DA MENINGITE EM MUNICÍPIO DA REGIÃO SUL DO BRASIL COM FOCO NA ETIOLOGIA MAIS COMUM
}

\section{EPIDEMIOLOGICAL SITUATION OF MENINGITIS IN THE SOUTHERN REGION OF BRAZIL MUNICIPALITY FOCUSING ON THE MOST COMMON ETIOLOGY}

\author{
Sara Naomi Shimabukuro ${ }^{1^{*}}$, Layane Sanches Fernandes ${ }^{1}$, Jorides Zoratto Neto ${ }^{1}$, \\ Kenji Rocha Nakahara ${ }^{1}$, Luciana Osorio Cavalli ${ }^{2}$ \\ ${ }^{1}$ Acadêmica do curso de Medicina do Centro Universitário FAG. ${ }^{2}$ Graduada em Medicina. Mestre em \\ Biociências e Saúde \\ *Autor Correspondente: snshimabukuro@minha.fag.edu.br, https://orcid.org/0000-0002-5655-0584
}

\section{RESUMO}

Introdução: a meningite consiste em uma doença endêmica no Brasil que apresenta surtos e epidemias ocasionais. Objetivo: avaliar o perfil epidemiológico da meningite em um município da Região Sul do Brasil. Métodos: estudo descritivo que analisou a situação epidemiológica da meningite em um município da Região Sul do Brasil com foco na etiologia mais comum. O estudo utilizou dados secundários obtidos a partir do Sistema de Informação de Agravos de Notificação do município de Cascavel-PR no período de 2008 a 2018. Resultados: os casos de meningite acometeram, em sua maioria, a raça branca e o sexo masculino. Houve maior frequência de etiologia asséptica e presença de quadro clínico com cefaleia, febre e vômitos. A complementação diagnóstica foi realizada por meio de exame quimiocitológico de líquor. A maioria dos pacientes evoluíram para alta hospitalar. Conclusão: apesar da escassez de estudos sobre a temática para a construção de um comparativo, os resultados em relação aos aspectos epidemiológicos foram consistentes com a literatura. A meningite acomete, de maneira geral, pessoas do sexo masculino e apresenta etiologia asséptica majoritariamente. É necessário, portanto, o enfoque aos grupos populacionais mais comumente acometidos pela meningite.

Palavras-chave: Meningite; Sistemas de Informação em Saúde; Epidemiologia.

\section{ABSTRACT}

Introduction: meningitis is an endemic disease in Brazil which presents occasional outbreaks of epidemics. Objective: to evaluate the epidemiological profile of meningitis in a municipality in the Southern Region of Brazil. Methods: a descriptive study of the meningitis epidemiological situation in the Southern Region of Brazil municipality focusing on the most common etiology. This study used secondary data from the Cascavel-PR Health Information Systems in the period of 2008 to 2018. Results: meningitis cases were mostly common in white and male groups. There was a higher frequency of aseptic etiology and clinical symptoms like headache, fever and vomit. The diagnostic complementation was made by chemocytological tests of cerebrospinal fluid. Most part of the patients progressed to discharge from hospital. Conclusion: despite the low number of studies about this subject to make a confrontation, the epidemiological aspects results were consistent to the literature. The meningitis occurs in the male group and it has aseptic etiology in the majority. Therefore it is necessary to focus on the population groups most commonly affected by meningitis.

Keywords: Meningitis; Health Information Systems; Epidemiology. 


\section{INTRODUÇÃO}

A meningite consiste no processo inflamatório das meninges por etiologia infecciosa ou não. Considerada uma doença endêmica no Brasil, apresenta surtos e epidemias ocasionais (BRASIL, 2019).

As ações de vigilância e controle da meningite são preconizadas pelo Ministério da Saúde. Os sintomáticos que se dirigem ao serviço ambulatorial ou hospitalar são submetidos à coleta de dados clínicos e epidemiológicos associada ao internamento e a procedimentos médico-hospitalares (PARANÁ, 2015).

O serviço deve preencher a Ficha de Investigação Epidemiológica e notificar a Vigilância Epidemiológica da Secretaria Municipal de Saúde, que, sequencialmente, repassa a informação à Regional de Saúde e essa, por sua vez, à Divisão de Vigilância das Doenças Transmissíveis da Secretaria de Estado da Saúde (Guia Rápido de Manejo Epidemiológico das Doenças Imunopreveníveis).

A notificação compulsória da meningite possibilitou informar a confirmação de 17.454 casos notificados no Brasil em 2018 (Sistema de Informação de Agravos de Notificação), apesar das medidas para a prevenção e o controle dessa doença propostas pela Organização Mundial de Saúde. Essas correspondem à imunização das pessoas e quimioprofilaxia aos contactantes (WHO, 2019).

No mesmo ano, 1.481 dos casos notificados confirmados evoluíram para óbito. A Região Sul ocupou o segundo lugar em relação à quantidade de notificações da doença (SINAN). O trabalho tem como objetivo uma análise da situação epidemiológica da meningite em um município da Região Sul do Brasil com foco na etiologia mais comum.

\section{METODOLOGIA}

Realizou-se um estudo descritivo na população de abrangência no âmbito da saúde do município de Cascavel-PR. O estudo utilizou dados secundários obtidos a partir do Sistema de Informação de Agravos de Notificação (Sinan) do local. Os dados consistiram nas notificações de meningite feitas no período de 2008 a 2018. Essas apresentaram uma limitação relacionada à qualidade dos dados disponibilizados devido ao modo como a ficha de notificação foi preenchida em alguns casos.

As informações relacionadas à identificação, ao diagnóstico e ao tratamento foram tabuladas em uma planilha do Microsoft Excel 2013. Além disso, a análise incluiu a frequência absoluta e relativa de acordo com o tipo de meningite e sexo, sendo que, nessa comparação, foram realizados testes de proporções que consideraram hipóteses bilaterais e assumiram nível se significância de $5 \%$. Essa análise, por sua vez, foi feita com o software estatístico R versão 3.52 (R Core Team).

O estudo respeitou as normas preconizadas pela Resolução 466/12 do Conselho Nacional de Saúde para a realização de pesquisas envolvendo seres humanos.

O projeto foi aprovado pelo Comitê de Ética em Pesquisa do Centro Universitário Assis Gurgacz (CEP-FAG) sob o parecer de no 08444918.7.0000.5219, sendo que a pesquisa foi autorizada pela Secretaria Municipal de Saúde do município.

\section{RESULTADOS}

No total, entre 2008 a 2018, foram notificados 591 casos de meningite no município de Cascavel-PR. Houve uma oscilação no número de infectados no 
decorrer de cada ano, sendo que o número máximo de notificações ocorreu em 2008 com 87 casos e o mínimo, por sua vez, em 2016 com 24.

Os casos de meningite estão concentrados nos segmentos de raça branca $(90,69 \%)$ e parda $(5,14 \%)$, em residentes de zona urbana $(96,44 \%)$ e, em relação ao nível de instrução, muitos casos notificados não informaram o grau de escolaridade. No entanto, entre os casos informados, a maior parte apresentava o ensino fundamental incompleto.

A maior proporção de casos ocorreu no sexo masculino com 358 notificações $(60,57 \%)$, sendo que, em 277 casos de meningite asséptica (etiologia mais frequente), cerca de $57,76 \%$ dos pacientes eram homens.

Dentre as pessoas que foram notificadas com Meningite dos tipos asséptica, não especificada, por outras bactérias, por Pneumococos e tuberculosa, pode-se afirmar que a incidência em homens é estatisticamente superior, comparado aos casos em mulheres ( $p$-valor $<0,05)$, conforme quadro 1.

Quadro 1. Número de notificações de pacientes com meningite e p-valor, referente ao teste de proporção, de acordo com o sexo. Cascavel-PR, 2008-2018.

\begin{tabular}{|c|c|c|c|}
\hline ETIOLOGIA & FEMININO & MASCULINO & P-VALOR \\
\hline ASSÉPTICA & 117 & 160 & 0,0116 \\
\hline OUTRA ETIOLOGIA & 12 & 17 & 0,4576 \\
\hline MENINGOCÓCICA & 7 & 5 & -- \\
\hline MENINGOCÓCICA COM & 3 & 3 & -- \\
MENINGOCOCCEMIA & & & 0,0288 \\
\hline NÁO ESPECIFICADA & 13 & 28 & -- \\
\hline POR HEMÓFILO & 1 & 1 & 0,0033 \\
\hline POR OUTRAS BACTÉRIAS & 53 & 89 & 0,0311 \\
\hline POR PNEUMOCOCOS & 9 & 22 & 0,0055 \\
\hline TUBERCULOSA & 1 & 12 & 0,6171 \\
\hline HIPÓTESE DESCARTADA & 16 & 20 & -- \\
\hline MENINGOCOCCEMIA & 1 & 1 & $\mathbf{0 , 0 0 2 3}$ \\
\hline TOTAL & $\mathbf{2 3 3}$ & $\mathbf{3 5 8}$ &
\end{tabular}

No entanto, na comparação da meningite asséptica no decorrer dos anos, observou-se, conforme Quadro 2, que a incidência da doença em homens é estatisticamente superior as mulheres apenas nos anos de 2009 e 2017 ( $p$ valor $<0,05)$. Muitos casos não puderem ser comparados via testes estatísticos devido ao baixo número de observações.

Quadro 2. Número de notificações de pacientes com meningite asséptica, de acordo com o sexo e o ano. Cascavel-PR, 2008-2018.

\begin{tabular}{|c|c|c|c|c|}
\hline ANO & FEMININO & MASCULINO & TOTAL & P-VALOR \\
\hline 2008 & $21(48,84 \%)$ & $22(51,16 \%)$ & 43 & 1,0000 \\
\hline 2009 & $7(25,00 \%)$ & $21(75,00 \%)$ & 28 & 0,0140 \\
\hline 2010 & $6(42,86 \%)$ & $8(57,14 \%)$ & 14 & -- \\
\hline 2011 & $10(52,63 \%)$ & $9(47,37 \%)$ & 19 & 1,0000 \\
\hline 2012 & $8(29,63 \%)$ & $19(70,37 \%)$ & 27 & 0,0543 \\
\hline 2013 & $16(53,33 \%)$ & $14(46,67 \%)$ & 30 & 0,8551 \\
\hline 2014 & $11(50,00 \%)$ & $11(50,00 \%)$ & 22 & 1,0000 \\
\hline 2015 & $10(52,63 \%)$ & $9(47,37 \%)$ & 19 & -- \\
\hline 2016 & $2(33,33 \%)$ & $4(66,67 \%)$ & 6 & -- \\
\hline 2017 & $8(28,57 \%)$ & $20(71,43 \%)$ & 28 & 0,0376 \\
\hline 2018 & $18(43,90 \%)$ & $23(56,10 \%)$ & 41 & 0,5322 \\
\hline $\mathbf{2 0 0 8 - 2 0 1 8}$ & $\mathbf{1 1 7}(\mathbf{4 2 , 2 4} \%)$ & $\mathbf{1 6 0 ( 5 7 , 7 6 \% )}$ & $\mathbf{2 7 7}$ & $\mathbf{0 , 0 1 1 6}$ \\
\hline
\end{tabular}


O quadro clínico apresentou majoritariamente cafaleia, febre e vômitos (Quadro 3), além de outros sintomas em 36,54\% dos casos. O exame físico, por sua vez, não apresentou, na maioria dos casos, rigidez de nunca, Kernig ou Brudzinski positivos, abaulamento de fontanela e petéquias (Quadro 4).

Quadro 3. Sintomatologia apresentada pelos pacientes de acordo com os casos notificados. Cascavel-PR, 2008-2018.

\begin{tabular}{|c|c|c|c|c|}
\hline SIM & $65,82 \%$ & $80,03 \%$ & $61,59 \%$ & $15,05 \%$ \\
\hline NÃO & $26,56 \%$ & $19,79 \%$ & $37,22 \%$ & $84,26 \%$ \\
\hline IGNORADO & $7,61 \%$ & $0,16 \%$ & $1,18 \%$ & $0,67 \%$ \\
\hline
\end{tabular}

Quadro 4. Exame físico apresentado de acordo com os casos notificados. Cascavel-PR, 2008-2018.

\begin{tabular}{|c|c|c|c|c|}
\hline PRESENÇA & $\begin{array}{c}\text { RIGIDEZ } \\
\text { DE NUCA }\end{array}$ & $\begin{array}{c}\text { KERNIG/ } \\
\text { BRUDZINSKI }\end{array}$ & $\begin{array}{c}\text { ABAULAMENTO DE } \\
\text { FONTANELA }\end{array}$ & PETÉQUIAS \\
\hline SIM & $31,13 \%$ & $6,09 \%$ & $6,26 \%$ & $3,21 \%$ \\
\hline NÂO & $67,00 \%$ & $91,53 \%$ & $92,21 \%$ & $95,93 \%$ \\
\hline
\end{tabular}

O diagnóstico foi complementado pela punção lombar em $97,46 \%$ das notificações com realização de quimiocitológico de líquor para confirmação em $66,48 \%$ dos mesmos. Ao todo, foram confirmados $93,90 \%$ dos casos.

A maioria dos pacientes evoluíram para alta (Quadro 5), sendo que houve $98,47 \%$ hospitalizações e $6,42 \%$ dos pacientes ficaram em coma.

Quadro 5. Evolução dos pacientes notificados como meningite. Cascavel-PR, 2008-2018.

\begin{tabular}{|c|c|}
\hline EVOLUÇÃO & $\%$ \\
\hline ALTA & 88,49 \\
\hline ÓBITO POR MENINGITE & 5,41 \\
\hline ÓBITO POR OUTRA CAUSA & 4,73 \\
\hline IGNORADO & 1,35 \\
\hline
\end{tabular}

\section{DISCUSSÃO}

A meningite é uma doença que independe das estações climáticas e, consequentemente, pode ocorrer durante todo o ano. Os avanços no diagnóstico e na vigilância epidemiológica promovida pelo Ministério da Saúde possibilitaram uma redução no número de casos da doença (RODRIGUES, 2015). No período estudado, houve uma oscilação no número de infectados no decorrer de cada ano, sendo que o número máximo de notificações ocorreu em 2008 com 87 casos e o mínimo, por sua vez, em 2016 com 24.

O estudo demonstrou uma concentração na raça branca em 90,69\% das notificações, sendo que o perfil epidemiológico dos casos de meningite é caracterizado majoritariamente por pessoas de tal segmento racial (DAZZI, ZATTI, BALDISSERA, 2014; GONÇALVES E SILVA, MEZAROBBA, 2018).

O perfil epidemiológico contempla, de maneira geral, o sexo masculino na incidência da doença (MONTEIRO et al, 2014; MORAIS et al, 2017; DA SILVA GOMES et al, 2019; DE LIMA FONTES, 2019) como pode-se visualizar em 60,57\% dos casos desse estudo. Além disso, a etiologia mais frequente consiste na meningite asséptica, sendo que cerca de $57,76 \%$ dos pacientes acometidos por essa causa eram homens (DAZZI, ZATTI, BALDISSERA, 2014; GONÇALVES E SILVA, MEZAROBBA, 2018; DE LIMA FONTES, 2019). O estudo permitiu inferir que a incidência da meningite asséptica em homens é estatisticamente superior as mulheres nos anos de 2009 e 2017 (p-valor<0,05). 
O quadro clínico caracteriza-se pela presença de cefaleia, febre e vômitos (CREPALDI et al, 2014), como foi demonstrado na pesquisa em conjunto com outros sintomas (36,54\%). O diagnóstico foi complementado com a realização de quimiocitológico de líquor, método diagnóstico mais utilizado (DAZZI, ZATTI, BALDISSERA, 2014; RODRIGUES, 2015; GONÇALVES E SILVA, MEZAROBBA, 2018; DA SILVA GOMES et al, 2019), para confirmação em $66,48 \%$ dos casos. A evolução para alta hospitalar é frequente (MONTEIRO et al, 2014; GONÇALVES E SILVA, MEZAROBBA, 2018; DA SILVA GOMES et al, 2019; DE LIMA FONTES, 2019) com ocorrência em $88,49 \%$ dos casos no estudo realizado.

O trabalho apresenta uma fortaleza baseada nos conceitos de prevenção e promoção da saúde, como descrito pela Medicina de Família e Comunidade/Saúde da Família. Além disso, os resultados desse estudo expõem a necessidade de maiores estudos sobre a temática na região Sul do Brasil visto que a construção de um comparativo foi dificultada pela escassez dos mesmos.

\section{CONSIDERAÇÕES FINAIS}

Os dados do Sinan demonstraram uma oscilação no número de infectados no decorrer de cada ano no município de Cascavel-PR, sendo que o número máximo de notificações ocorreu em 2008 com 87 casos e o mínimo, por sua vez, em 2016 com 24.

Os casos de meningite acometeram majoritariamente a raça branca e o sexo masculino com maior frequência de etiologia asséptica. O quadro clínico apresentou, de maneira geral, cefaleia, febre e vômitos e complementação diagnóstica por meio da realização de exame quimiocitológico de líquor. A maioria dos pacientes evoluíram para alta hospitalar.

É necessário o enfoque aos grupos populacionais mais comumente acometidos pela meningite. As Equipes de Atenção Básica e de Vigilância em Saúde devem desenvolver um trabalho integrado em prol da promoção da saúde e da prevenção de doenças, bem como promover assistência ao conhecimento para identificação dessa doença de notificação compulsória. Tais sistemas de apoio são importantes para reduzir a incidência e promover o diagnóstico da meningite.

\section{REFERÊNCIAS}

BRASIL. Ministério da Saúde. Saúde de A a Z: Meningites. Disponível em: <http://www.saude.gov.br/saude-de-a-z/meningites>. Acesso em: 26 jun. 2019.

BRASIL. Ministério da Saúde. Secretaria de Vigilância em Saúde. Sistema de Informação de Agravos de Notificação, Sinan Net. Disponível em: $<$ http://tabnet.datasus.gov.br/cgi/tabcgi.exe?sinannet/cnv/meninbr.def>. Acesso em: 26 jun. 2019.

CREPALDI, P. I. S.; DIAS, C. A. R.; LARIUCCI, R.; SILVA, R. M. V.; GIANELLA, J. C. B.; SANVITO, W. L. et al. Estudo epidemiológico e clínico sobre meningite em adultos no setor de emergência em São Paulo. Arquivos Médicos dos Hospitais e da Faculdade de Ciências Médicas da Santa Casa de São Paulo, v. 59, n. 1, p. 1-6, 2014. 
DA SILVA GOMES, L.; DA SILVA PASSOS, B. V.; DA SILVA AZEVEDO, P. S.; JÚNIOR, F. T. D. S. S.; SAMPAIO, L. S.; MATOS, L. F. L et al. Aspectos epidemiológicos das meningites virais no estado do Piauí no período de 2007 a 2017. Revista Eletrônica Acervo Saúde, v. 11, n. 10, p. e433-e433, 2019. https://doi.org/10.25248/reas.e433.2019

DAZZI, M. C.; ZATTI, C. A.; BALDISSERA, R. Perfil dos Casos de Meningites Ocorridas no Brasil de 2009 a 2012. Revista UNINGÁ Review, v. 19, n. 3, p. 33-36, 2014.

DE LIMA FONTES, F. L. Aspectos epidemiológicos da meningite no estado do Piauí: 2007 a 2017. Revista Ciência \& Saberes - FACEMA, v. 4. n. 4, 2019.

GONÇALVES E SILVA, H. C.; MEZAROBBA, N. Meningite no Brasil em 2015: O panorama da atualidade. Arquivos Catarinenses de Medicina, v. 47, n. 1, p. 34-46, 2018.

MONTEIRO, L. F.; FRASSON, M. Z.; TREVISOL, D. J.; SCHUELTER-TREVISOL, F. Vigilância clínico-epidemiológica das meningites em um hospital do sul de Santa Catarina, no período entre 2007 a 2013. Arquivos Catarinenses de Medicina, v. 4, n. 43, p. 24-29, 2014.

MORAIS, J. M. R.; DA ROCHA, L. D. H.; COSTA, T. P.; DE SOUSA, M. N. A. Retrato da meningite em Salvador-BA: análise do período entre 2011-2015. Ciência \& Desenvolvimento - Revista Eletrônica da FAINOR, v. 10, n. 1, 2017.

PARANÁ (Estado). Secretaria de Estado da Saúde. Centro de Epidemiologia. Guia Rápido de Manejo Epidemiológico das Doenças Imunopreveníveis. 2015.

R Core Team. R: A language and environment for statistical computing. R Foundation for Statistical Computing, Vienna, Austria, 2018. Disponível em: <https://www.Rproject.org/>

RODRIGUES, E. M. B. Meningite: perfil epidemiológico da doença no Brasil nos anos de 2007 a 2013. Brasília, 2015, 16p. Trabalho de Conclusão de Curso, Faculdade de Ciências da Educação e Saúde, Centro Universitário de Brasília.

World Health Organization, 2019.2 Disponível em: <https://www.who.int/emergencies/diseases/meningitis/en/>. Acesso em 26 jun. 2019. 